\title{
Depression deserves better treatment
}

\author{
Kirsten Patrick MBBCh DA
}

See also salon article on page 1112 and at www.cmaj.ca/lookup/doi/10.1503/cmaj.141126, and news article at www.cmaj.ca/lookup /doi/10.1503/cmaj.109-4897

$\mathrm{P}$ ublic perceptions about depression are rife with bafflement and false beliefs. Just look at the public reaction on digital media to the recent suicide of Robin Williams. I was surprised to encounter repeatedly the opinion that depression is a failing ("get a better attitude," "be positive") and is easily fixed with the right quality of religious faith. The majority wondered, "Why did he suffer alone?" Why, indeed. Why do so many? Why do we persist in stigmatizing and neglecting depression despite its high prevalence?

Based on disability-adjusted life-years, unipolar depressive disorder is ranked ninth in the most recent estimates from the World Health Organization (WHO) Global Burden of Disease 2010 study. ${ }^{1}$ On a global scale, its burden is greater than that of diabetes or tuberculosis. TB and malaria, with lesser global burden, get official WHO global public health "days." Not depression. In high-income countries, only ischemic heart disease and stroke cause more disability than unipolar depression. If the amount of money donated to various charities is a way of gauging the relative importance attributed to medical causes, then depression goes down the list like a lead balloon: in Canada, when ranked by donation amount, not one mental-health charity cracks the top-10 list. ${ }^{2}$

The burden of depression arises not just from the high cost of treating this condition, but also from the reduced functional ability of those affected, which leads to their reduced productivity. Effects on children and other loved ones can also be enduring and serious. Patients with chronic depression are at increased risk of comorbid illness, and incident depression also frequently complicates chronic physical conditions. Furthermore, depression affects younger people as frequently as it does older people, and it tends to recur throughout a person's life.

The fact that we don't have widely accessible and effective treatments for depression is a serious problem. Recent evidence suggests that antidepressants alone don't work much better than placebo for many people. ${ }^{3}$ Behavioural therapies, particularly cognitive behavioural therapy, are moderately effective with effects that endure, but they are frequently difficult to access, rely on properly trained personnel for delivery, take time to work and have high attrition rates. ${ }^{4}$ Exercise is consistently shown to be effective in both treating and preventing depression, but motivation is a barrier in those for whom performing simple personal functions may be difficult. Creative interventions that use physical activity in group settings with buddy support can help, with the added bonus of mandated social interaction. Neuromodulation treatments were reviewed in $C M A J$ last year. ${ }^{5}$ Electroconvulsive therapy, itself highly stigmatized, is one, but there are other promising neuromodulatory interventions with evident short- to medium-term effectiveness. Unfortunately, few can access these treatments in Canada and the cost-effectiveness of using them in the Canadian setting has not been investigated. A combination of approaches - and ideally a tailored approach - may work best. Patients are discovering this for themselves and are sharing their experiences on online forums and blogs. However, policy lags behind.

We are not alone in asking why mental illness is such a low priority worldwide, in spite of coordinated efforts to destigmatize mental illness in recent years. The concept of "no health without mental health" has gained wide traction among health professionals worldwide, yet progress in improving identification of depressed patients and ensuring swift access to the right treatment has been slow. Mitigating the effects of depression on those close to the affected person continues to receive scant attention.

In Canada, we need to stop treating depression as a Cinderella disease. We need to fund research that can make a difference to people with depression. The federal government must make a commitment to improving mental-health infrastructure nationwide. Health practitioners ought to be able to connect patients identified as depressed with adequate support and appropriate treatment without delay. Community programs can play an important role in filling the support and treatment-delay gaps, and these programs should be boosted. We need to scale up training for behavioural therapists. Exercise interventions are cheap to run and effective, but they are currently underused and require funding support. Most neuromodular therapies are still classified as experimental; facilities and trained staff are rare, and relative expense is high. Yet, we should not limit these effective treatments to those whose depression is intractable and prolonged. Although substantial resources are needed, the high cost of depression to individuals, families and society justifies the expense. But such resources will only receive priority if we all decide to pay more positive attention to depression.

\section{References}

1. Estimates for 2000-2012: Disease burden. Geneva (Switzerland): World Health Organization; 2014. Available: www.who.int/healthinfo/global_burden_disease /estimates/en/index2.html (accessed 2014 Sept. 1).

2. Canada's top 10 largest charities. Toronto: Charity Intelligence Canada; 2013. Available: www.charityintelligence.ca/canadas-top-10-largest-charities (accessed 2014 Sept. 1).

3. Fournier JC, DeRubeis RJ, Hollon SD et al. Antidepressant drug effects and depression severity: a patient-level meta-analysis. JAMA 2010;303:47-53.

4. Hofmann SG, Asnaani A, Vonk IJ, et al. The efficacy of cognitive behavioral therapy: a review of meta-analyses. Cognit Ther Res 2012;36:427-40.

5. Lipsman N, Sankar T, Downar J, et al. Neuromodulation for treatment-refractory major depressive disorder. CMAJ 2014;186:33-9.

Competing interests: See www.cmaj.ca/site/misc/cmaj_staff.xhtml

Affiliation: Kirsten Patrick is Deputy Editor, CMAJ

Correspondence to: $C M A J$ editor, pubs@cmaj.ca

CMAJ 2014. DOI:10.1503/cmaj.141128 